\title{
Chronic Obstructive Pulmonary Disease in Primary Care by Dr. David Bellamy and Rachel Booker
}

\author{
D Keeley
}

Title

Chronic Obstructive

Pulmonary Disease in

Primary Care

\section{Authors}

Dr. David Bellamy and

Rachel Booker

\section{Publisher:}

Class Health London

ISBN 1-872362-95-8

Book Review by:

Duncan Keeley

General Practitioner

Thame

Oxon OX $93 \mathrm{JZ}$

Prim Care Respir J 2001:

10(2);52
Chronic obstructive pulmonary disease is a cinderella condition if ever there was one. We tend to be pessimistic about our ability to help much, and to provide less well organised and co-ordinated care for this devastating condition than we do for asthma, diabetes or hypertension. David Bellamy - a respected GP expert in respiratory disease, and Rachel Booker - a nurse and COPD course leader at the NARTC in Warwick, have got together and written an excellent practical text to help us improve matters. Their accounts of the pathology, assessment and management of COPD are unfailingly crisp, businesslike and helpful. The material is presented in a way that will serve both to introduce the subject to newcomers clearly and comprehensibly, and to challenge those who already know some of it to organise their understanding to greater practical effect for the optimisation of patient care. A clutch of important practical topics are dealt with along the way - the role and the interpretation of spirometry, a scheme for therapeutic trials, how to help with smoking cessation (minus zyban), nebuliser therapy, the use of oxygen.

We are rightly reminded from time to time of the enormous importance of having and conveying a positive attitude to the management of COPD. We should not confine our attention to the often limited effect we can have on lung function with our treatments, but do everything we can to help patients live better with the problems. This means being sensitive to the profound psychological and social impact of a disease which can seriously incapacitate a person before it even makes them look particularly ill. This means thinking about mood, about family dynamics, about benefits, about rehabilitation programmes and not just about bronchodilators and steroids.

So far as bronchodilators and steroids are concerned, their role and limitations are concisely and sensibly discussed. Here as elsewhere we are not treated to an exhaustive and exhausting comprehensive evidence base - but key references are listed at the chapter ends. In the case of the vexed question of whether inhaled steroids help, the recent major trials are presented with a useful degree of detail as to the patients, interventions and outcomes so as to allow a better assessment of what these studies mean.

A large part of the art of doing a good job on writing a book like this is hitting the right balance between an impressive display of knowledge and the succinct and practical help desired by the non-enthusiast. In this book there are some places where we could have had a bit more - for example more details of social security benefits and more about terminal care. But on the whole I think the authors have got the balance right.

\section{Managing Chronic Obstructive Pulmonary Disease in Primary Care by Antony Crockett}

\section{Levy}

\section{Title}

Managing Chronic

Obstructive Pulmonary

Disease in Primary Care 144 pp, refs 227

\section{Authors}

Antony Crockett

Publisher:

Blackwell Science 2000.

ISBN 063205696

\section{Book Review by:}

Mark Levy

marklevy@animalswild.c

om or Mark.Levy@,

gp-E84663.nhs.uk

Prim Care Respir J 2001: 10(2);52
Dr Antony Crockett is a primary care physician with a special interest in respiratory disease. His book is 144 pages long and written under the following chapter headings: Introduction; COPD the disease; Management of COPD; The primary care practice; Audit; COPD the future and Further information which includes details on relevant publications, organisations and respiratory pharmaceutical companies.

In the introduction the author makes a good case for correctly diagnosing, monitoring, educating and prescribing for patients suffering from COPD. Next in,'COPD the disease', he devotes 25 pages to the epidemiology, pathogenesis, pathophysiology and natural history of COPD. The rest of this chapter is devoted to clinical features and assessment of COPD; including tests of 'function' such as an outline description of the six minute walk test, the MRC scale of dyspnoea, oxygen cost and quality of life as well as a brief mention of spirometry without going into this subject in depth. This chapter concludes with the differential diagnosis from asthma, pulmonary oedema, angina, infection and lung cancer.

The chapter on management provides an overview of the various non-pharmacological options available and recommended for COPD including smoking cessation strategies. Pharmacological management is covered in this chapter, with perhaps a disproportionate focus on the use of inhaled steroids, though the author does conclude the use of these drugs should be limited to those who demonstrate symptomatic or functional response (including quality of life) and to those with severe COPD. Management of acute exacerbations is well described in this chapter.

Dr Crockett then describes some of the practicalities of running a COPD service in primary care followed by some examples of medical audit on the subject. The final chapter considers possible future improvements in COPD care, including the way in which care is delivered and some of the drug trials in progress. 